\title{
Nachlässe in der Württembergischen Landesbibliothek
}

Zum Bestand der Württembergischen Landesbibliothek zählt eine Reihe von Nachlässen unterschiedlicher Persönlichkeiten'. Bei den Nachlassern handelt es sich in erster Linie um Personen mit Bezug zu Württemberg. Viele dieser Personen weisen eine Verbindung zu Stuttgart oder der näheren Umgebung auf. Darunter befindet sich ein nicht geringer Anteil an Nachlassern mit Musikbezug. Diese Nachlässe sind vor allem im Laufe der letzten Jahrzehnte in den Bestand gekommen. Größtenteils handelt es sich bei den Nachlässen der Landesbibliothek um Schenkungen aus Familienbesitz, einzelne Nachlässe wurden aber auch angekauft. Die Nachlässe unterscheiden sich voneinander erheblich in der Größe des Umfangs und darüber hinaus in der Art ihrer Zusammensetzung. Die in der WLB vorhandenen Nachlässe sind auf der Website der Handschriftensammlung nachgewiesen. Handelt es sich um einen Nachlass mit Bezug zur Musik, wird er auf der Website der Musiksammlung mit detaillierteren Informationen aufgelistet. Zuständig für die Benutzung der Nachlässe ist die Handschriftensammlung. Eine Benutzung ist grundsätzlich nur nach vorheriger Anfrage möglich. Es können sich dabei allerdings Einschränkungen ergeben, die unter anderem mit dem Erschließungsstand und den Persönlichkeitsrechten in Zusammenhang stehen.

\section{Von der Art, der Beschaffenheit und dem Umgang mit Nachlässen}

Der Zustand des Nachlassmaterials, wie es in die Landesbibliothek kommt, kann von sehr unterschiedlicher Beschaffenheit sein: Manchmal ist ein Nachlass von Familienangehörigen bereits vorsortiert oder gar schon in beschriftete (aber i.d.R. nicht säurefreie) Mappen verpackt. Gelegentlich findet man auch eine Vorsortierung der brieflichen Korrespondenz vor. In anderen Fällen sind die Spuren der Zeit deutlich sichtbar und man sieht, dass die Unterlagen lange keine Behandlung oder Benutzung mehr erfahren haben. In allen Fällen muss das Material neu verpackt werden. Das geschieht im Zusammenhang mit der Verzeichnung.
Dabei wird darauf geachtet, dass die Neuverpackung mit säurefreien Mappen und Archivboxen erfolgt. Eine solche Verpackung ist in vielen Fällen mit einem nicht geringen Aufwand verbunden: Zunächst muss eine geeignete Verpackung gefunden werden. Besteht ein Nachlass aus einzelnen (Brief-)Konvoluten, dann gilt es diese sinnvoll in Einzelmappen einzulegen. Dabei muss jede Mappe und jeder erste Brief eines Konvoluts mit einer Signatur versehen werden. Mehrere dieser Mappen werden anschließend in die passende Archivbox gelegt. Auch diese muss ordnungsgemäß eine Beschriftung über die zu beinhaltenden Einzelmappen erhalten.

Ebenso unterschiedlich wie der Zustand des Materials ist der Umfang eines Nachlasses. Manchmal handelt es sich nur um zwei bis drei Archivboxen. In anderen Fällen wird deutlich mehr Platz im Magazin benötigt, um die Materialien unterzubringen. Bei vielen Nachlässen handelt es sich in erster Linie um Briefkorrespondenzen, die in nicht wenigen Fällen durch weiteres Material in Form von Manuskripten, gedruckten Zeitschriftenartikeln oder Büchern ergänzt werden. Häufig sind dies Aufzeichnungen zu eigenen Werken der Nachlasser. Gelegentlich birgt ein Nachlass allerdings auch Notizsammlungen auf Makulaturpapier, die in der Erschließung sehr aufwändig sein können, denn jedes Blatt sollte gezählt und damit foliiert werden. Aber auch ganze Konvolute eigenen

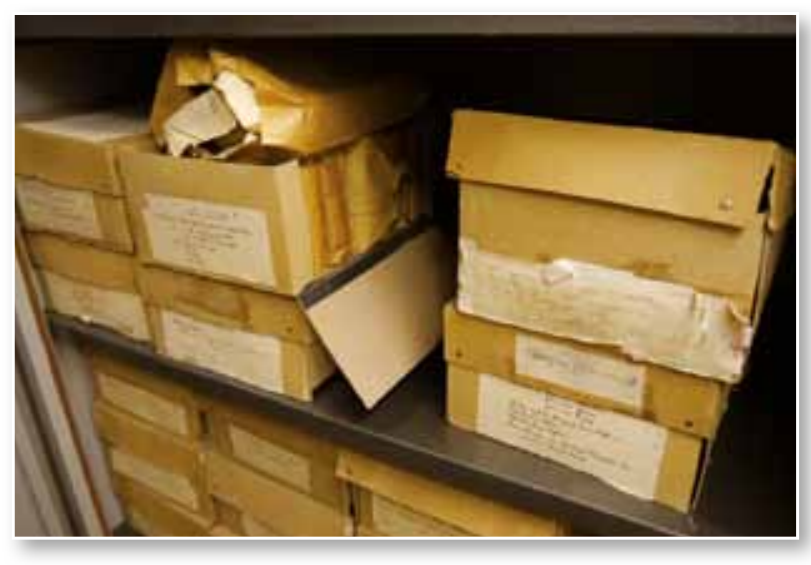

Abb. 1: Nachlass Christoph König vor der Erschließung und Neuverpackung
(1) Wenn im Folgenden von Nachlässen die Rede ist, sind die zahlreichen Fälle, bei denen es sich lediglich um Teilnachlässe handelt, mit eingeschlossen. 


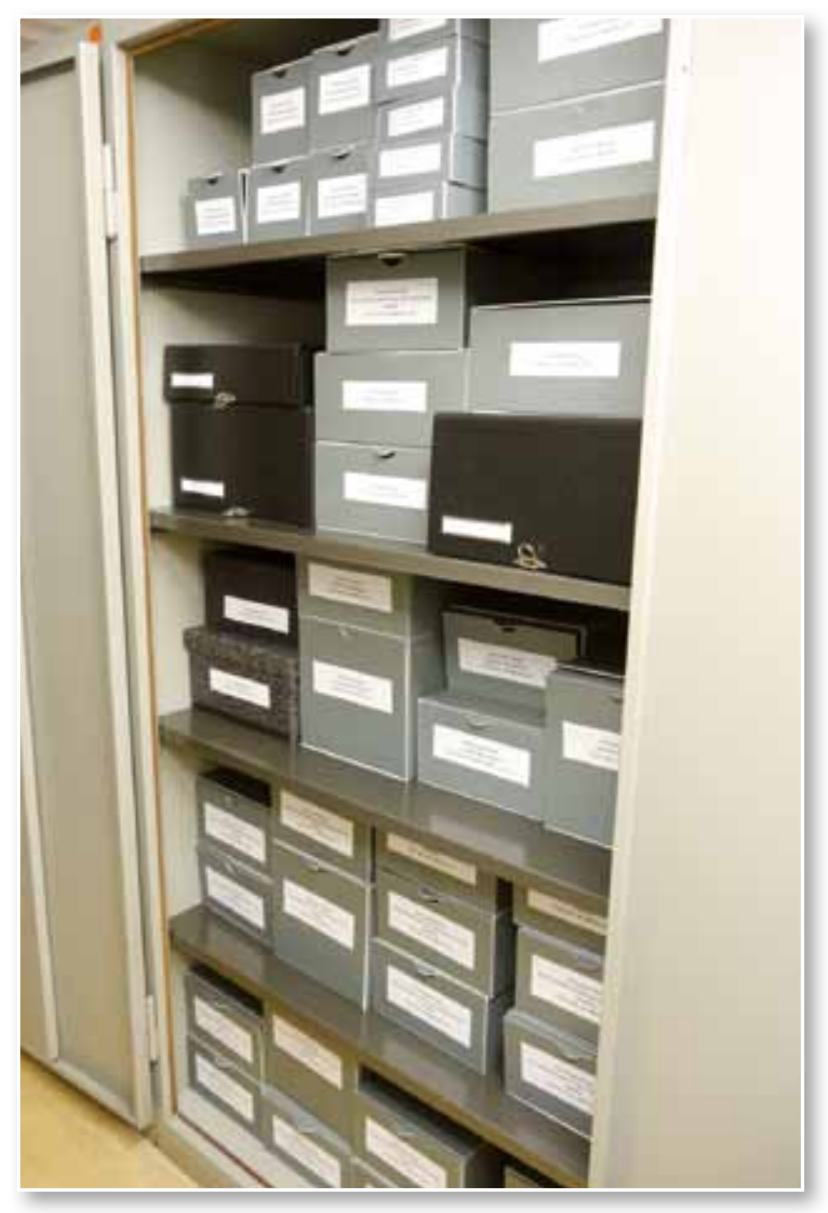

Abb. 2: Nachlass Christoph König nach der Erschließung und Neuverpackung

Schrifttums der Nachlasser sind vorhanden. Dazu zählen Vortrags- oder Buchmanuskripte genauso wie Handexemplare der Autoren, die Notizen und Anmerkungen enthalten.

\section{Erschließung}

In der Regel kommen Nachlässe in unerschlossenem Zustand in die Bibliothek. In einigen wenigen Fällen ist bereits eine Erschließung vorhanden. Ein Beispiel hierfür stellt der (Teil-)Nachlass Immanuel Faißt dar: er enthält Notenmanuskripte, die teilweise von Faißt selbst geschrieben wurden. Diesen Manuskripten ist ein Katalog in Form von handschriftlich beschriebenen Katalogkarten mit dem Titel "Katalog der Musikwerke in Abschrift aus dem Nachlaße Immanuel Faißts" beigefügt. Dieser Katalog stammt aus der Musikhochschule Stuttgart, über welche der Nachlass in die Landesbibliothek gekommen ist. ${ }^{2}$

Die Erschließung eines Nachlasses kann sich je nach Art und Beschaffenheit unterschiedlich aufwändig gestalten. In einigen Fällen können umfangreiche Provenienz- und Personenrecherchen notwendig sein. In der Regel erfolgt die ErschlieBung zunächst durch eine Sichtung und Vorsortierung des Materials, was mit der Vergabe einer allgemeinen Bestandssignatur und Signaturen der Unterbestände einhergeht. Im Anschluss daran folgt die Feinerschließung. Das beinhaltet beispielsweise im Fall einer Briefkorrespondenz die Identifizierung der Absender, eine Sortierung der Absender in alphabetischer Reihenfolge sowie die Vergabe einer Individualsignatur für jeden Absender.

Eine Verzeichnung von Nachlässen findet in der Württembergischen Landesbibliothek und auch in vielen anderen Bibliotheken bzw. Einrichtungen Deutschlands mit Hilfe der Datenbank Kalliope (Verbundkatalog für Autographen und Nachlässe) statt. Die Datenbank ist online unter http://kalliopeverbund.info frei zugänglich. Hier kann der Bestand mit seiner Gliederung in Unterbestände angelegt und angesehen werden. Erfasst werden in Kalliope vor allem Briefkorrespondenzen, aber auch die Eingabe weiterer Nachlassmaterialen ist möglich. So finden z. B. auch Fotos, Urkunden oder Medaillen eine Erwähnung, die immer wieder Teil von Nachlässen sind. Im musikalischen Kontext setzen sich die Nachlässe häufig aus Korrespondenz und Noten bzw. Kompositionen zusammen. Beides wird in Kalliope verzeichnet.

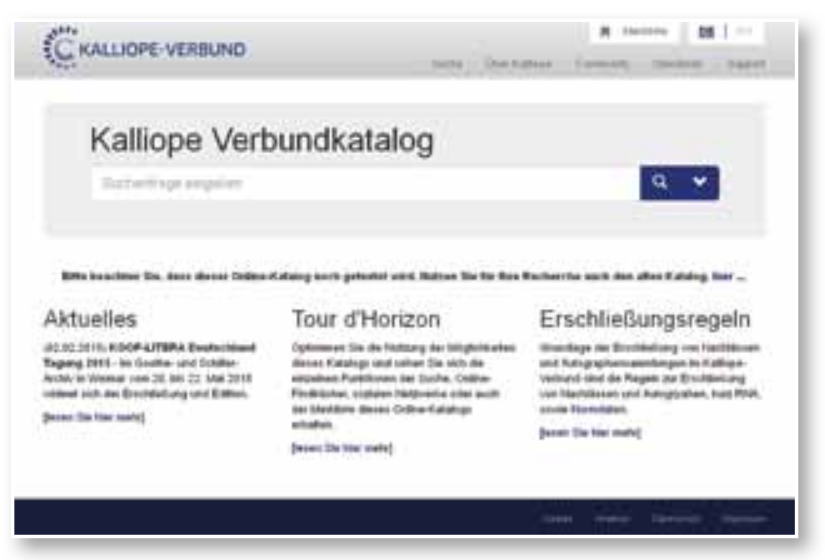

Abb. 3: Kalliope Verbundkatalog

\section{Neu erschlossene Nachlässe}

Im letzten Jahr wurden einige Nachlässe der Landesbibliothek erschlossen. Im Fokus standen dabei Nachlässe aus dem Bereich der Musik. Bisherige größere Erschließungsprojekte der Landesbibliothek aus diesem Bereich umfassten vor einigen Jahren bereits die Nachlässe Hugo Herrmann (1896-1967) (Cod. mus. II fol. 345, Cod. hist. qt. 
759) und Helmut Bornefeld ${ }^{3}$ (1906-1990) (Cod. hist. qt. 758, Cod. mus. II fol. 342).

Von diesen neu erschlossenen Nachlässen soll im Folgenden eine Auswahl vorgestellt werden.

Der umfangreichste der in jüngster Zeit erschlossenen Nachlässe ist der Nachlass des württembergischen Hymnologen Christoph König (18431913) aus Eberdingen (Cod. hist. qt. 802). ${ }^{4}$ Dieser Nachlass wurde 1968 von Nachfahren Königs der Landesbibliothek als Geschenk übergeben. Seither konnte er nur unter Schwierigkeiten benutzt werden. Königs Anliegen war es, "die schönsten und wertvollsten Lieder aus der vorreformatorischen Zeit bis zur Neuzeit (bis etwa 1900) im Originaltext" zu sammeln. Besondere Sorgfalt wollte er auch der "Verfasser- und Quellenangabe widmen" ${ }^{5}$. Vor allem für die Lieder des 18. und 19. Jahrhunderts musste er in der Regel eigene Forschungen anstellen. Seine Korrespondenz (s.u.), die er mit bekannten deutschen Hymnologen und diversen Bibliotheken, besonders in Stuttgart, München, Leipzig, Hamburg, Wernigerode, Greifswald und Göttingen unterhielt, ist im Nachlass überliefert.

König hat ein hymnologisches Lexikon verfasst, das Originaltexte von ca. 9.000 Kirchenliedern beinhalten sollte (zusammen mit einem 10.00011.000 Lieder umfassenden kritischen Apparat). Dieses Werk konnte zu seinen Lebzeiten aus Kostengründen nicht gedruckt werden. Obwohl er bereit gewesen wäre, für den Druck seine wertvolle Sammlung hymnologischer Fachliteratur und Gesangbücher zu veräußern, war ihm eine Umsetzung des Planes nicht möglich. Ein Verwandter, der Lehrer August Brunner, bemühte sich im Ruhestand um die Fortführung und Beendigung des Lexikons. Von Brunner befindet sich ein Kryptonachlass im Nachlass König. Er erstellte drei hilfreiche Register (Dichter nach Zeitperioden, Alphabetisches Gesamtverzeichnis der Lieder, Alphabetisches Dichterverzeichnis), die eine bessere Benutzung von Königs Material möglich machen. Die Register liegen in Form eines Typoskripts vor. Hinzu kommen Aufzeichnungen und Vorarbeiten sowie einige Briefe mit Bezug zur Hymnologie oder zu Christoph König. Die umfangreiche hymnologische Bibliothek und die Gesangbuchsammlung von Christoph König kamen mit dem Nachlass 1968 in die WLB. Gesangbücher und hymnologische Fachliteratur wurden getrennt vom Nachlass im Musikmagazin aufgestellt. Der Nachlass selbst umfasst neben den Aufzeichnungen zum hymnologischen Lexikon eine Korrespondenz mit ca. 130 Absendern, bei denen es sich in erster Linie um Personen mit Bezug zum Thema Kirchenlied handelt (Hymnologen, Theologen etc.). Ebenso dazu gehören Korrespondenzen mit Bibliotheken, da König immer wieder auf der Suche nach spezieller Fachliteratur gewesen ist, in der er Informationen zu den einzelnen Liedern finden konnte. Der Nachlass ist durch ein Verzeichnis erschlossen und in seiner Struktur auch in Kalliope erfasst. Im Rahmen der Erschließung wurde das gesamte Material neu verpackt. Eine Neuverpackung in säurefreies Material war dringend notwendig. Stellenweise war die Pappe durch den Säuregehalt verbräunt, ein Großteil des Materials war spröde und schmutzig, wodurch das Papier nur weiter angegriffen wurde. Zum Teil waren „Zettelpakete" in Zeitungspapier eingewickelt, was zur Folge hatte, dass Druckerschwärze an das Papier gelangt ist. Die Neuverpackung trägt nun zum langfristigen Erhalt dieses Nachlasses bei, der jetzt der hymnologischen Forschung zur Verfügung steht.

Ein weiterer Nachlass aus dem Bereich Musik ist der des Pforzheimer Musikdirektors Theodor Röhmeyer (1869-1944). Hierbei handelt es sich lediglich um einen Teilnachlass (Cod. hist. qt. 788), weitere Bestände aus seinem Gesamtnachlass befinden sich im Stadtarchiv Pforzheim und der Staatsbibliothek zu Berlin (Musikabteilung). Den Teil, den die WLB besitzt, hat man in den Jahren 1969-1976 von Röhmeyers Witwe Hannah Röhmeyer angekauft. Er besteht mit Ausnahme weniger Notenbände ausschließlich aus Korrespondenz, die sich in drei Bereiche untergliedern lässt: Briefe an Theodor Röhmeyer, Briefe an den in Würzburg wirkenden Komponisten Cyrill Kistler (1848-1907) und Briefe anderer (nicht an Theodor Röhmeyer).

Der Nachlass des Stuttgarter Musikschriftstellers und -kritikers Karl Grunsky (1871-1943) kam erst in

(3) Das Bestandsverzeichnis wurde erstellt von Martin, Jörg: Der Komponist Helmut Bornefeld (1906 - 1990): Verzeichnis seines Nachlasses in der Württembergischen Landesbibliothek. Augsburg 2006 (2 Bände).

(4) Vgl. die Ausführungen von Gottfried Mälzer zu Christoph König und dem Erwerb der Sammlung für die WLB in: Blätter für Württembergische Kirchengeschichte 68/69 (1968/69), S. 644-646.

(5) August Brunner in dem Vorwort zu seinem selbst erstellten Register zu Königs Lexikon. 
jüngster Zeit in den Besitz der WLB (Cod. hist. qt. 806). Er besteht aus einer umfangreichen Korrespondenz mit ca. 360 zeitgenössischen Personen aus dem Bereich des Musiklebens sowie Notizen und Aufschrieben zu diversen Themen der Musik und Typoskripten. In der Korrespondenz befinden sich auch ein Briefwechsel mit dem Musikverlag C. F. Peters in Leipzig, bei dem es um die Herausgabe von Bruckners Sinfonien durch Grunsky geht, sowie einige Briefe aus Haus Wahnfried in Bayreuth.

Die Nachlässe von zwei in Stuttgart wirkenden Komponisten sowie einer Komponistin sind ebenfalls Teil der Sammlungen der WLB: Der Nachlass des Stuttgarter Organisten und Komponisten Matthäus Koch (1862-1933) umfasst neben Noten auch eigene philosophisch-religiöse Texte und zahlreiche Gedichte (Cod. hist. qt. 799). Koch war königlicher Musikdirektor und Organist an der Friedenskirche in Stuttgart von 1893 bis 1933. Wilhelm Kempff (1895-1991) war ein berühmter Pianist, Organist und Komponist. Zeitweise war er als Nachfolger Max von Pauers Leiter der Musikhochschule Stuttgart. Von inm hat die Landesbibliothek Fotos sowie einige wenige Notendrucke, die aus dem Besitz seiner Schülerin Charlotte Poerschke stammen (Cod. hist. qt. 801). Die Drucke sind überwiegend mit einer handschriftlichen Widmung Kempffs an Poerschke versehen und sind Teil der Musiksammlung. Bei Wilhelm Kempff (zuvor bei Max Pauer) hatte die Stuttgarter Komponistin Hilda KocherKlein (1894-1933) studiert und Klavierunterricht genommen. Sie stammt aus einer Musikerfamilie (Eltern: Franz Klein, Solobratschist, Emilie Klein, geb. Schuler, Pianistin), die Teil des Stuttgarter Musiklebens war. ${ }^{6}$ Kocher-Kleins Nachlass (Cod. hist. qt. 803) beinhaltet ihre Kompositionen in Form von handschriftlichen Manuskripten zusammen mit einem Werkverzeichnis, das ihre Freundin Rosemarie Neundorf erstellt hat. Die Werke umfassen über 100 Opuszahlen und weitere Werke ohne Opuszahl.

Aus dem Besitz der Familien von Brand und Bergmann besitzt die Württembergische Landesbibliothek einen Teilnachlass (Cod. hist. qt. 805). Er beinhaltet in erster Linie (handschriftliche) No-

(6) Zur Biographie vgl. Friedel, Claudia: Komponierende Frauen im Dritten Reich: Versuch einer Rekonstruktion von Lebensrealität und herrschendem Frauenbild. Münster u.a.: Lit, 1995, S. $310 f f$.

(7) Vgl. zur Ballettsammlung von Serge Leslie und Doris Niles: Trost, Vera (Hrsg.): Dance \& dancers, Stuttgart Ballet. Heidelberg: Braus, 1997, S. 11-22. tenmanuskripte aus Familienbesitz. Die Noten stammen mit großer Wahrscheinlichkeit aus dem Besitz der Alice von Brand, geb. Bergmann (1874 bis nach 1936). Sie war die Tochter des Chirurgen Ernst von Bergmann (1836-1907) und war verheiratet mit Wilhelm Freiherr von Brand, mit dem sie in Stuttgart lebte. Die Manuskripte tragen in einigen Fällen Besitzeinträge und Widmungen verschiedener Familienmitglieder. Aus handschriftlichen Einträgen lässt sich erkennen, dass die Noten offenbar im privaten Musikunterricht verwendet worden sind.

Die Landesbibliothek besitzt auch den Nachlass des US-amerikanischen Tänzerehepaars Serge Leslie und Doris Niles (Cod. hist. qt. 798). Sie haben darüber hinaus ihre bedeutende Sammlung tanztheoretischer Bücher der WLB vermacht. ${ }^{7}$ Ihre Büchersammlung, die als „Ballettsammlung" ge-

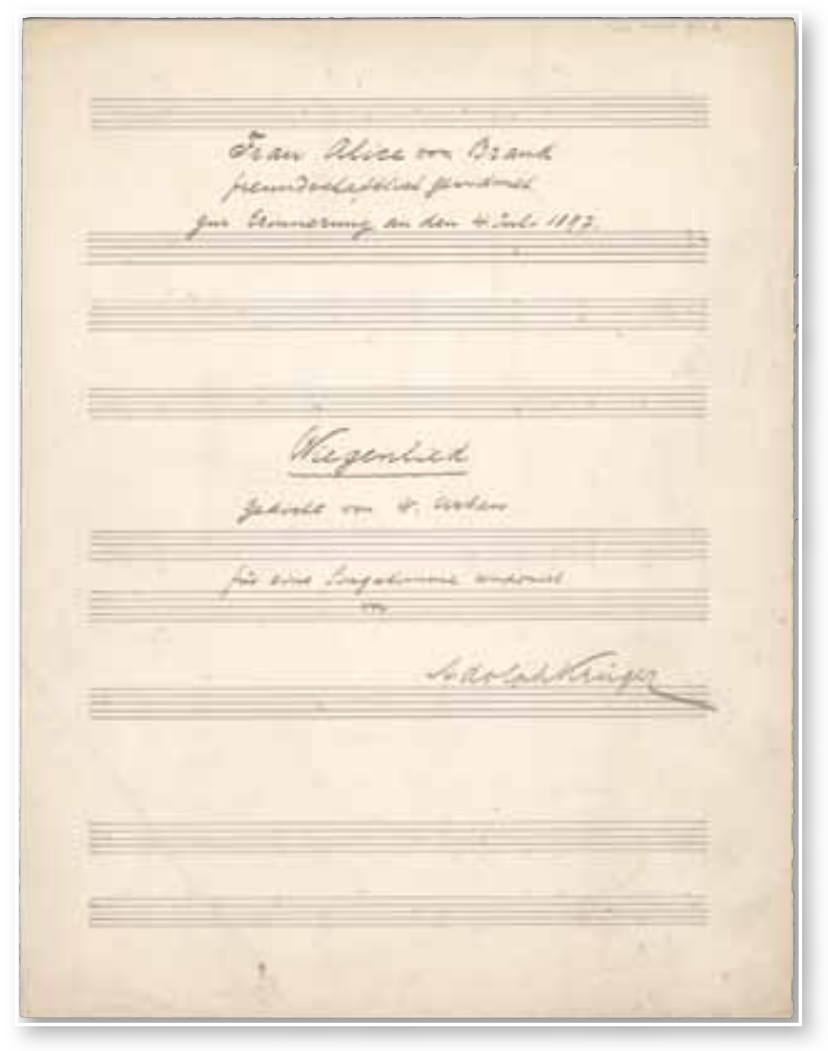

Abb. 4: Widmung des Komponisten Adolph Krüger an Alice Brand (Cod. hist. qt. 805,8): Frau Alice von Brand freundschaftlich gewidmet, zur Erinnerung an den 4. Juli 1897

sondert aufbewahrt wird, wurde bereits vor längerer Zeit in den Bestand integriert. Einzelne Bände waren immer wieder Teil von Ausstellungen an der WLB. Der Nachlass beinhaltet Briefe von ca. 40 Absendern, die in den meisten Fällen an Leslie und Niles gemeinsam gerichtet sind. Die Sammlung wird bereichert durch Fotos, die sich sowohl in der 
Korrespondenz als auch besonders in drei Alben (betitelt mit "Artists and Friends") befinden. Vor allem die Fotos der Alben sind immer wieder mit Widmungen versehen. Ferner enthält der Nachlass drei Konvolute mit diversen Zeitungsausschnitten und weiteren zeitgenössischen Materialien, die Auftritte von Doris Niles dokumentieren und illustrieren.

Ein Nachlass, der weder Musik noch Ballett zum Thema hat, ist der Nachlass „Familienarchiv Pfaff/Moll“ (Cod. hist. qt. 791). Dieser Nachlass enthält Briefe, Dokumente und Schrifttum von verschiedenen Familienmitgliedern der miteinander verwandten Familien Pfaff und Moll. Das Familienarchiv Moll umfasst dabei allerdings einen verhältnismäßig kleinen Teil. Der älteste Brief aus 46 dem Familienarchiv Pfaff geht bis in die Mitte des 16. Jahrhunderts zurück. Der Brief ist auf das Jahr 1532 datiert und stammt von Johannes Pfaff aus Cannstatt, der an den Bürgermeister von Esslingen, Bernhard Mayer, genannt Motzbeck, schreibt. Die übrigen Briefe und Materialien stammen mehrheitlich aus dem 18. und 19. Jahrhundert, einzelne Briefe datieren noch in das 17. Jahrhundert. Bei der Korrespondenz handelt es sich größtenteils um familieninterne Briefwechsel. Von dem Stuttgarter Archivar Carl Friedrich Pfaff (1764-1836) haben sich aber auch ein kleines Briefkonvolut aus Korrespondenz mit dem Haus Württemberg (Herzöge Carl Eugen, Ludwig Eugen, Friedrich II.) und eines mit dienstlicher Korrespondenz erhalten. Der Hauptbestandteil der brieflichen Korrespondenz stammt von Friedrich Burkhard Pfaff (1738-1817), dem Archivar Carl Friedrich Pfaff (1764-1836), dem Lehrer Karl Pfaff (1795-1866) und dem Gymnasialprofessor Karl Hermann Siegfried Pfaff (18271907). Der andere Teil des Nachlasses ist nicht weniger umfangreich und beinhaltet Dokumente zur Familienchronik, Schrifttum (besonders Manuskripte von Karl Siegfried Pfaff) und persönliche Aufzeichnungen einzelner Familienmitglieder, sowie Reden zu Hochzeiten und Beerdigungen, Lebensbeschreibungen und Taufregister einzelner Familienmitglieder. Ferner enthält das Material auch einen Stammbaum der Familie Pfaff sowie einen längeren Briefwechsel zur Hillerschen Familienstiftung.
Die Fülle der sich in der Landesbibliothek befindenden Nachlässe spiegelt in ihrer Vielfalt das künstlerisch-intellektuelle Leben in Württemberg (vor allem in Stuttgart) in intensiver Form wider und ermöglicht der Forschung Einblicke in Korrespondenz und detailliert festgehaltene Sachverhalte von Persönlichkeiten besonders mit regionaler Bedeutung.

Maria Gramlich 